\title{
Ising spin glass by the transfer matrix approach
}

\author{
R. F. S. Andrade* and E. Nogueira, Jr. ${ }^{\dagger}$ \\ Instituto de Física, Universidade Federal da Bahia, Campus da Federação, CEP 40210-340, Salvador, Bahia, Brazil \\ Sérgio Coutinho \\ Laboratório de Física Teórica e Computacional, Departamento de Física, Universidade Federal de Pernambuco, CEP 50670-901, \\ Recife, Pernambuco, Brazil \\ (Received 3 March 2003; revised manuscript received 5 May 2003; published 25 September 2003)
}

\begin{abstract}
The short-range Ising spin glass on the diamond hierarchical lattice is investigated via a transfer-matrixbased method. An exact set of discrete maps leads to all thermodynamical functions for any finite lattice, from which the thermodynamic limit can be obtained. The method, which encompasses the random choice of the coupling constants between neighboring sites, has been applied to both Gaussian and bimodal probability distributions. Results for the thermodynamic potentials of the model defined on lattices with fractal dimension $d_{f}=2,2.58 \ldots$ and 3 , below and above the estimated lower critical dimension $\left(d_{l} \sim 2.5\right)$, are discussed and fully analyzed. Typical Schottky-like profiles are observed in the temperature behavior of the specific heat for both distributions in all lattices. Finite residual entropy is found to persist for the bimodal distribution case. When $d_{f}>d_{l}$ and for large number of iterations the correlation length $\xi$ increases exponentially in a wide temperature interval. The divergence of $\xi$ at a finite temperature $T_{c}$ associated with the spin-glass phase transition is investigated within an approximate scheme. The numerical values for $T_{c}$ and $\nu$ are brought in comparison with those previously obtained by other methods.
\end{abstract}

DOI: 10.1103/PhysRevB.68.104523

PACS number(s): 75.10.Nr, 05.50.+q, 64.60.Fr

\section{INTRODUCTION}

In the past thirty years, despite the huge amount of work that has been put into the investigation of the prototypical models of disordered systems, namely the spin-glass (SG) and the random-field Ising models, basic questions concerning their condensed phase and critical behavior are still far from being settled. ${ }^{1-4}$

Concerning SG the general properties of the condensed phase are described by two conflicting scenarios regarding the multiplicity of pure states at $T=0$ : the one coming from the so-called droplet framework, which is appropriated to describe models with short-range interactions ("realistic" models), and the replica symmetry breaking (RSB) one, which arises from the mean-field solution of the infiniterange coupling model. In the latter, the free-energy landscape in the configurational space is composed of a high number of pure states arranged onto an ultrametric structure of valleys separated by high-energy barriers, ${ }^{5}$ while in the former the landscape should be trivial, that is, composed of just two single pure states related by global spin inversion symmetry. ${ }^{6}$ Whether such a RSB picture prevails in real (short-range models) systems is a very controversial question that remains unanswered. $^{7}$ To attack this problem many models/ methodologies have been considered so far. For instance, the search of the ground-state multiplicity of the short-range Ising spin-glass model (SRISGM) has been an object of interest of many authors, who developed sophisticated numerical methodologies for direct counting of ground states, such as mappings into combinatory optimization problems, ${ }^{8}$ biological motivated algorithms, ${ }^{9}$ and Monte Carlo multicanonical techniques. ${ }^{10}$

However, regarding exact solvable models for the SRISGM there are only a few ones, mostly beyond but still within mean-field approximation such as the one defined on the Cayley tree lattice. ${ }^{11}$ On the other hand, several approximated methods and numerical procedures have been widely used to explore the nature of the spin-glass condensed phase such as power-series expansions, ${ }^{12}$ Monte Carlo methods, ${ }^{13}$ and real-space renormalization group. ${ }^{14}$ The latter can be viewed either as an approximation for the model defined on Bravais lattices or as an exact solvable procedure for the model defined on hierarchical lattices. Actually these lattices were conceived to furnish the model such that the real-space Migdal-Kadanoff renormalization group (MKRG) transformation ${ }^{15,16}$ becomes realizable, that is exactly solvable. ${ }^{17,18}$ For instance, the pure Ising model on diamond hierarchical lattices has been proved to be exactly solved within the MKRG scheme. ${ }^{18,19}$ For random systems, such as spin glasses, this scheme gives results comparable to other approaches (Monte Carlo simulations, for example) for threedimensional lattices. ${ }^{20}$ It seems that the randomness of couplings and/or fields washes out the effects caused by the lack of translational invariance and by the concomitantly high inhomogeneous site coordination-number distribution of the hierarchical lattices. Some important results have been obtained for the SRISGM defined on hierarchical lattices $(\mathrm{HL})^{21-24}$ as well as for deterministic aperiodic models. ${ }^{25}$

In this work, we investigate the properties of the SRISGM on diamond hierarchical lattices (hereafter DHL's) with a quite distinct method than the previous ones, namely the transfer-matrix (TM) method. The proposed procedure amounts to directly evaluate the thermodynamic properties, free energy, and its derivatives, together with the correlation length $\xi$, for particular realizations of the model. Here we consider the SRISGM defined in terms of two probability distribution functions for the coupling constants, the $\delta$-bimodal and the Gaussian ones. Mean values, for both 
probability distributions, are obtained by collecting data for a large number of distinct realizations within the same procedure. We explore three lattices with the same geometry but with different fractal dimensions $d_{f}=2,2.58$ and 3, which lie around the presumed value, $d_{l}=2.5$, of the lower critical dimension for the occurrence of the SG condensed phase. ${ }^{26-28}$ Like MKRG, the TM method also profits from the exact scale invariance of the lattice. It has been used to analyze periodic and aperiodic Ising models on the same lattices, leading to a very precise evaluation of all relevant thermodynamic functions as function of the temperature, including the critical exponents and log-periodic oscillations in the neighborhood of the critical point. ${ }^{29}$

The rest of the work is so organized: in the following section we describe the procedure and derive the recurrence maps for the free energy, entropy, specific heat, and correlation length for the lattice with $d_{f}=2$. In Sec. III, we discuss the behavior for the free energy and its derivatives. Special emphasis is laid on the convergence of the results in the thermodynamic limit and on residual entropy for the SG with bimodal distribution. In Sec. IV, we discuss the behavior of the correlation length and questions related to its convergence in the thermodynamic limit, which require the introduction of an approximate scheme. Finally, we finish with Sec. V with some concluding remarks and perspectives.

\section{TRANSFER MATRIX FOR SG ON DHL'S}

General properties of random transfer matrices have been quite extensively investigated and used in the analysis of disordered systems. ${ }^{30}$ In the present study, we extend a TMbased formalism developed to analyze periodic and aperiodic spin systems on DHL's to encompass fully disordered spin systems, which are formally described by the following Hamiltonian

$$
H=-\sum_{(i j)} J_{i j} \sigma_{i} \sigma_{j}
$$

where $\sigma_{i}= \pm 1$ are Ising variables, the coupling constants $\left\{J_{i j}\right\}$ are quenched random variables following a welldefined probability distribution function $P\left(J_{i j}\right)$, and the sum applies to nearest-neighbor pairs of spins on a DHL.

The lattice is recursively generated, starting from a single bond linking the two root sites, which correspond to the DHL $G=0$ generation. The lattice is build by replacing each bond of the previous generation by a set of $p$ parallel branches, each one consisting of a chain of $b$ bonds with $b-1$ inner sites as shown in Fig. 1 for the case $p=b=2$. After many steps, this procedure generates a higher-order self-similar two roots graph, whose fractal dimension is $d_{f}$ $=(\ln b p) /(\ln b)$. In the present work, we limit ourselves to the case $b=2$ DHL's.

The basic idea of this approach is to write down the systems partition functions for any DHL generation $G$ in terms of a $2 \times 2 \mathrm{TM} U_{G}$ that contains the effect of all configurations mediated by the coupling constants $J_{i j}$ between the two root sites $\left(r_{1}\right.$ and $\left.r_{2}\right)$ in that generation $G$. Due to self-similar symmetry of the lattice, it is straightforward to derive ex-

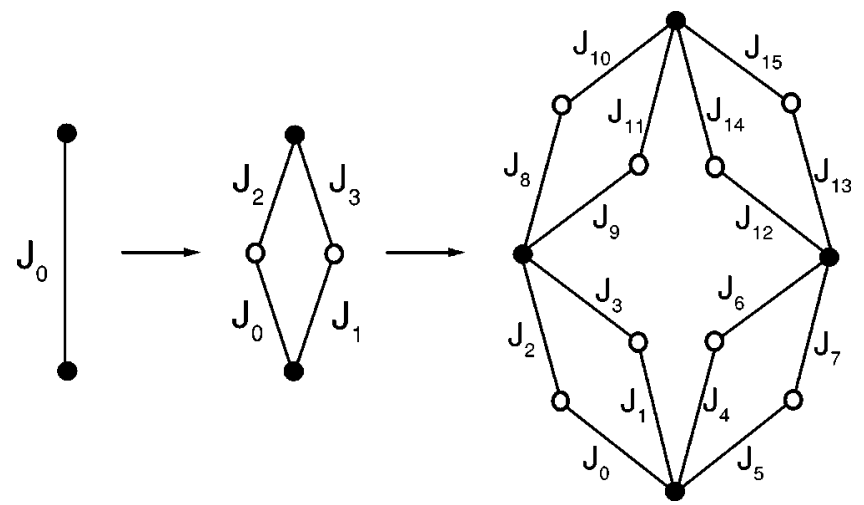

FIG. 1. The first three steps of the inflation process of an Ising SG on the DHL's with $p=q=2$. Each bond $J_{i}$ is independently chosen from the probability distribution. Each new generation may be constructed by connecting to the former one three replicas furnished with random coupling constants.

pressions relating the eigenvalues $\eta$ and $\epsilon$ of two subsequent generations, i.e., $\eta_{G+1}=\eta_{G+1}\left(\eta_{G}, \epsilon_{G}\right)$ and $\epsilon_{G+1}$ $=\epsilon_{G+1}\left(\eta_{G}, \epsilon_{G}\right)$. Such maps are conveniently written in terms of the free energy $f_{G}$ and correlation length $\xi_{G}$ defined by

$$
\begin{gathered}
f_{G}=-N_{G}^{-1} T \ln \eta_{G}, \\
\xi_{G}=\frac{M_{G}}{\ln \left(\eta_{G} / \epsilon_{G}\right)},
\end{gathered}
$$

where $\eta_{G}$ indicates the largest eigenvalues of $U_{G}$, and $N_{G}$ and $M_{G}$ are, respectively, for the number of spins and the lattice size, that is, the length of the shortest path connecting the two root sites for a given $G$-DHL. Note that Eq. (2), which goes along the usual definition of the free energy per spin for a system with periodic boundary conditions in the thermodynamical limit, is the correct form for a $G$-DHL, up to a term of the order of $T \ln 2 / N_{G} \rightarrow 0$ as $N_{G}$ grows. Therefore the numerical iteration of these maps leads to the thermodynamic properties of the model for any $G$ and as far as $N_{G} \sim 2^{3 G}$ assumes sufficiently large values to the numerically exact values in the thermodynamic limit.

Let $J_{0}$ be the coupling constant chosen at random from a probability distribution $P\left(J_{i j}\right)$ for the interaction between the spins at the root sites $r_{1}$ and $r_{2}$. It can be accounted for by the $2 \times 2 \mathrm{TM}$

$$
U_{G=0, k=0}=\left[\begin{array}{cc}
a_{0,0} & b_{0,0} \\
b_{0,0} & a_{0,0}
\end{array}\right],
$$

where $a_{0,0}=\exp \left(J_{0} / T\right)=b_{0,0}^{-1}$. The first subscript used to characterize $U$ refers to the generation, and the second one to the chosen value $J_{0}$ produced by $P\left(J_{i j}\right)$.

For $G=1$, the interaction between $r_{1}$ and $r_{2}$ is described by a $2 \times 2$ matrix $U_{1,0}$, with only two distinct matrix elements, arranged in the same way as $U_{0,0} . U_{1,0}$ can be expressed as

$$
U_{1,0}=Q_{0,0} Q_{0, p}^{t} .
$$


The $2 \times 2^{p}$ TM $Q_{0,0}$ describes the interactions between the spin at $r_{1}$ and those at the $p$ new inner sites, while $Q_{0, p}^{t}$, the transpose of $Q_{0, p}$, describes the interaction between the $p$ new spins and the one at $r_{2}$. For uniform systems, $U_{1,0}$ $=U_{1}$ and $Q_{0,0}=Q_{0, p}=Q_{0}$ depend on just one coupling constant $J_{0}$. The $Q_{0}$ matrix elements can be written in terms of those of $U_{0,0}=U_{0}$ :

$$
\begin{gathered}
\left(Q_{0}\right)_{i, l}=\left(U_{0}\right)_{i, j_{1}}\left(U_{0}\right)_{i, j_{2}} \ldots\left(U_{0}\right)_{i, j_{p}}, \quad l=1, \ldots, 2^{p}, \\
j_{s}=1,2, \forall s=1, \ldots, p,
\end{gathered}
$$

where the lexicographic order is used to map the $2^{p}$ elements of the set $\left\{j_{s}\right\} \rightarrow l$. With the help of Eqs. (5) and (6) an explicit expression for $U_{1}$ can be easily derived.

In a disordered system, $Q_{0,0}$ and $Q_{0, p}$ are functions of $p$ independent values of coupling constants $J_{i j}$. Let us relabel these constants, randomly produced by the distribution $P\left(J_{i j}\right)$, as $J_{l}$. Let also the interactions described by $Q_{0,0}$ be those with $l=0,1, \ldots, p-1$, while those with $l=p, \ldots, 2 p$ -1 refer to the interactions described by $Q_{0, p}$. The interaction $J_{0}$, present in the $G=0$-DHL, is incorporated into the $G=1$-DHL, requiring the random choice of only $2 p-1$ values for the new coupling constants. To each one of them we associate a transfer matrix $U_{0, l}$ defined in the same way as Eq. (4), which are used for the evaluation of $Q_{0,0}, Q_{0, p}$, and $U_{1,0}$. For instance, in the case $p=2$, the elements of $U_{1,0}$ are given by

$$
\begin{aligned}
a_{1,0}= & a_{0,0} a_{0,1} a_{0,2} a_{0,3}+a_{0,0} b_{0,1} a_{0,2} b_{0,3}+b_{0,0} a_{0,1} b_{0,2} a_{0,3} \\
& +b_{0,0} b_{0,1} b_{0,2} b_{0,3}, \\
b_{1,0}= & a_{0,0} a_{0,1} b_{0,2} b_{0,3}+a_{0,0} b_{0,1} b_{0,2} a_{0,3}+b_{0,0} a_{0,1} a_{0,2} b_{0,3} \\
& +b_{0,0} b_{0,1} a_{0,2} a_{0,3} .
\end{aligned}
$$

With the help of Eq. (7), we rewrite Eqs. (2) and (3) as

$$
\begin{gathered}
f_{1,0}=\frac{N_{0}}{N_{1}}\left(f_{0,0}+f_{0,1}+f_{0,2}+f_{0,3}\right) \\
+\frac{T}{N_{1}}\left\{\ln 2-\ln \left[1+z_{0,0} z_{0,1} z_{0,2} z_{0,3}\right]\right\}, \\
\xi_{1,0}=\frac{M_{1}}{M_{0}} \frac{\xi_{0,0} \xi_{0,2}}{\xi_{0,0}+\xi_{0,2}}\left\{1+\frac{\xi_{0,0} \xi_{0,2}}{\xi_{0,0}+\xi_{0,2}} \frac{1}{M_{0}} \ln \left[1+z_{0,0} z_{0,1} z_{0,2} z_{0,3}\right]\right. \\
\left.-\ln \left[1+z_{0,0}^{-1} z_{0,1} z_{0,2}^{-1} z_{0,3}\right]\right\}^{-1},
\end{gathered}
$$

where

$$
z_{0, l}=\frac{\epsilon_{0, l}}{\eta_{0, l}}, \quad N_{0}=2, \quad N_{1}=4, \quad M_{0}=1, \quad M_{1}=2 .
$$

The exact geometrical scale invariance of the lattice leads to the same recurrence maps for any generation $(G+1)$-DHL in terms of the corresponding quantities for the $G$-DHL's. So, the general recurrence maps are obtained by

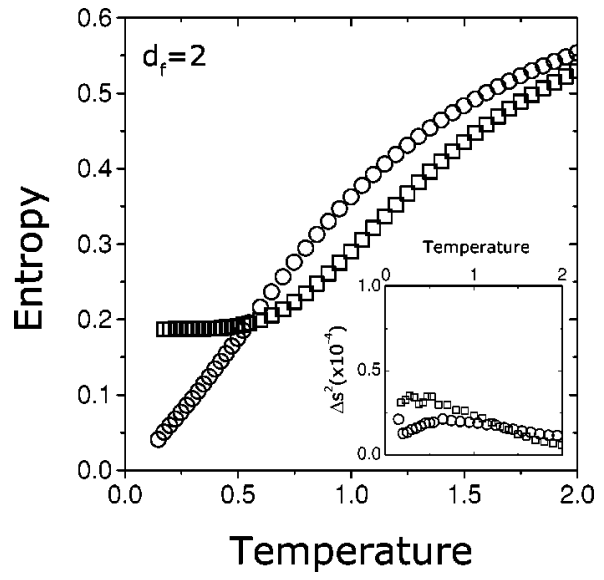

FIG. 2. Average entropy (50 samples) versus temperature for $P_{G}$ (circles) and $P_{b}$ (squares) when $d_{f}=2$ lattice $(p=2)$, indicating the presence of residual entropy for the $\delta$ bimodal probability distribution. Data dispersion is very small as shown in the inset.

replacing the subindices 0 and 1 by $G$ and $G+1$, respectively, in the above maps (8), with $N_{G}=2\left(2+4^{G}\right) / 3$ and $M_{G}=2^{G}$.

The thermodynamic properties of the model can be obtained by a numerical procedure based on Eq. (8). It consists of performing individual realizations of the disorder, by the recursive use of Eq. (8), where $J_{l}$ are randomly obtained from the distribution $P\left(J_{i j}\right)$.

In the process of evaluating $f_{G+1,0}$ and $\xi_{G+1,0}$ for increasing values of $G$, we observe that a $(G+1)$-DHL is built up of $2 p$ independent $G$-DHL's. As we keep the results $f_{G, 0}$ and $\xi_{G, 0}$ obtained for the first $G$-DHL, we have to evaluate only $2 p-1$ independent $G$-DHL's. However, this requires the independent evaluation of $2 p(G-1)$-DHL's for each of them, and so on. Thus, the central processing unit (CPU) time required to evaluate the properties of a lattice for a given value $G$ increases exponentially with a factor $2 p$. This is not surprising as the number of individually randomly chosen bonds, which must be individually accounted for, increases exponentially with $G$ as $(2 p)^{G}$.

This situation is quite different from that obtained for uniform (deterministic aperiodic) models, where the $(G+1)$-DHL is built up of $2 p$ copies of the same $G$-DHL (limited set of distinct $G$-DHL's). Thus, the CPU time increases only linearly with $G$, and results with a numerical precision of up to 16 significant digits (what can be understood as a numerical thermodynamic limit) can be easily achieved. ${ }^{29}$ Although the present results are as precise as those obtained for disordered systems by other methods, they can hardly be obtained with the same precision as for uniform systems due to CPU time restrictions.

Expressions similar to Eq. (8), valid for $p=3$ and 4 and used to obtain results discussed in the following sections, are written in the Appendix A. The above maps can be used as starting points for a larger set of maps of the derivatives of $f_{G}$ with respect to the temperature $T$, $\partial f_{G+1} / \partial T$ and $\partial^{2} f_{G+1} / \partial T^{2}$. They can be obtained in a straightforward way, leading to the entropy and specific heat of the system. The corresponding maps for Eq. (8) are written in the Appendix B. 


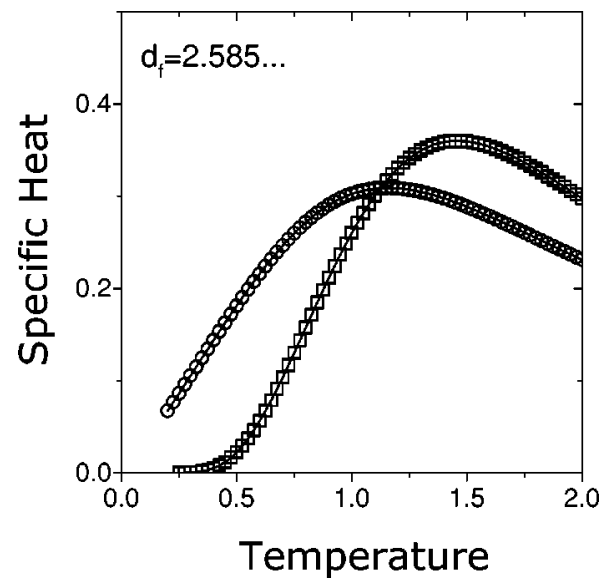

FIG. 3. Plots of the average specific heat for $d_{f}=2.585 \ldots$ lattice $(p=3)$ versus temperature for the Gaussian $\left(P_{G}\right)$ and the $\delta$-bimodal $\left(P_{b}\right)$ distributions (solid lines). Results for fifteen single realizations of the distribution are marked with circles $\left(P_{G}\right)$ and squares $\left(P_{b}\right)$, respectively. Individual points can hardly be distinguished within the used scale.

\section{FREE ENERGY AND ITS DERIVATIVES}

The iteration of Eqs. (8), (9) and (A1)-(A4), subject to initial conditions expressed by Boltzmann weights, leads to temperature dependent numerical values for the free energy and correlation length for individual realizations of $\mathrm{SG}$ on DHL's with $p=2,3$ and 4 , for $G=0, \ldots, G_{\max }$. The procedure can be extended, by the inclusion of maps (B1)-(B4) and their counterpart for $p=3$ and 4 , to evaluate the entropy and specific heat. In this section, we present the results for the free energy and its derivatives, which were obtained for the models defined in terms of the Gaussian,

$$
P_{G}\left(J_{i j}\right)=\sqrt{\alpha / \pi} \exp \left(-\alpha J_{i j}^{2}\right),
$$

and bimodal,

$$
P_{b}\left(J_{i j}\right)=\left[\delta\left(J_{i j}-1\right)+\delta\left(J_{i j}+1\right)\right] / 2,
$$

probability distributions for the couplings $\left\{J_{i j}\right\}$, where $\alpha$ $=1 / 2 J^{2}, J=1$ being the standard deviation. We considered $G_{\max }=10,9,8$, respectively, for $p=2,3,4$. This corresponds to $\sim 10^{6}-1.6 \times 10^{7}$ randomly chosen bonds for each realization. The results are based on samples of up to 400 distinct realizations (never less than 50) for each value of temperature, and were performed mainly on a 16 -node $800-$ $\mathrm{MHz}$ PentiumIII-cluster.

The results are summarized in Figs. 2-4. In Fig. 2, we show the sample averaged entropy as a function of the temperature, for both $P_{G}$ and $P_{b}$, and $p=2$. In the inset of Fig. 2 , we also show the temperature dependent dispersion to the

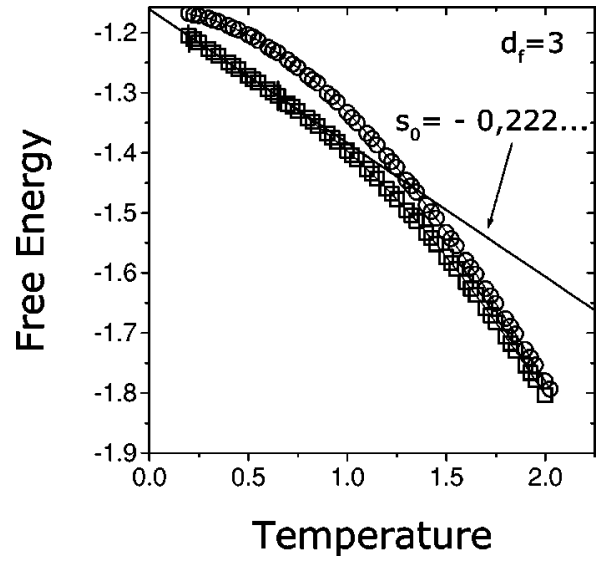

FIG. 4. Plots of the free energy for $d_{f}=3$ lattice $(p=4)$ versus temperature for the Gaussian $\left(P_{G}\right)$ and the $\delta$-bimodal $\left(P_{b}\right)$ distributions (solid lines). Results for 15 single realizations of the distribution are marked with circles $\left(P_{G}\right)$ and squares $\left(P_{b}\right)$, respectively. Individual points can hardly be distinguished within the used scale. The finite slope in the plot for $P_{b}$ is indicative of residual entropy.

mean value of the entropy. For the same distributions, we draw the curves for temperature dependence of the specific heat and the free energy in Figs. 3 and 4 for lattices with $(p=3)$ and $(p=4)$, respectively. Similar curves for other values of $p$, not shown, look like them.

All plots drawn in Figs. 2-4 are limited to values of $T$ $>0.15-0.25$. For both distributions, the Boltzmann weights become very large for smaller values of $T$, leading to numerical overflows. The very low values for the dispersion in Fig. 2 reflect the high precise values for all obtained quantities. In fact, within the used vertical scales, the points corresponding to each individual realization can hardly be distinguished from those shown in the averaged curves in Figs. 3 and 4.

At low temperatures, the entropy has rather different behavior for $P_{G}$ and $P_{b}$. For the first distribution, it goes linearly to zero as $T \rightarrow 0$, while a finite residual entropy builds up for the bimodal distribution. This is an expected behavior since the sites belonging to the last generation of a DHL have an even number of bonds and therefore, with the bimodal distribution the net field on these sites can be exactly zero, which corresponds to a twofold ground-state degeneracy per such sites. The same behavior is not expected for the continuous valued $P_{G} \cdot{ }^{14,22,31}$

For the three different values of $p$, the residual entropy can be extrapolated from the finite temperature data leading to values shown in Table I, which are compared with those obtained from a counting procedure for the average number of ground states ${ }^{22}$ for the same models. Tests on the conver-

TABLE I. Values for the residual entropy for the SRISGM with $\delta$-bimodal probability distributions of coupling constants.

\begin{tabular}{lccc}
\hline \hline & $d_{f}=2.0(p=2)$ & $d_{f}=2.585 \ldots(p=3)$ & $d_{f}=3.0(p=4)$ \\
\hline Present work & $0.1870 \pm 0.0006$ & $0.1977 \pm 0.0003$ & $0.219 \pm 0.001$ \\
Ref. 22 & $0.18227 \pm 0.00004$ & $0.162299 \pm 0.00002$ & $0.20842 \pm 0.00003$ \\
\hline \hline
\end{tabular}




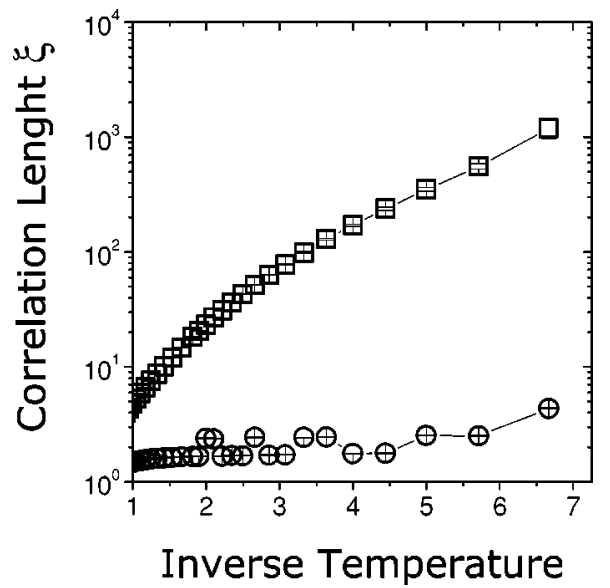

FIG. 5. Plots of the average correlation length (log scale) for both probability distributions versus the inverse of the temperature for the $d_{f}=2$ lattice $(p=2)$. Error bars indicate that dispersion is very small for $P_{b}$, while its values become significant in the lowtemperature region for $P_{G}$. For $T \rightarrow 0$, observe clear indications that $\xi$ (a) remains finite for $P_{b}$ (b) diverges weaker than $\exp (1 / T)$ for $P_{G}$.

gence of the present results, performed by increasing the value of $G_{\max }$ for all values of $p$, confirm the precision up to three significant digits.

All curves for the specific heat display, as the one shown in Fig. 3, a typical Schottky round profile with a smooth maximum. No qualitative distinction is observed from the one at $p=2$ to those at larger values of $p$ where phase transitions are expected to occur. For the free energy, we notice qualitative changes in the low-temperature region, between the curves produced by the different distributions. Those for $P_{b}$ have a constant nonzero slope, the value of which equals to $s_{0}$.

\section{CORRELATION LENGTH AND PHASE TRANSITION}

Results obtained for the correlation length $\xi$ upon iteration of Eqs. (8), (9) and (A1)-(A4) deserve a more detailed discussion due to a much slower convergence of the results with respect to the value of $G$, in comparison with those for the free energy and its derivatives. As already mentioned, in the investigations carried out for both homogeneous and deterministic aperiodic systems, the maps have been iterated until numerical convergence of all 16-digits variables. This does not represent a big problem, as the CPU time increases linearly with $G$. A value for $G_{\max }$ is not explicitly required, as the iteration proceeds until the convergence condition is met. However, it is possible to note that, depending on $T, 40-80$ iterations are required for that convergence. It is also possible to observe that $f$ always converges at smaller values of $G$ than $\xi$. Thus, a slower convergence for $\xi$ in the present case is also expected. This situation is illustrated in Fig. 5. In this figure, we draw the sample averaged and ten individual realizations for $\xi$ as function of $1 / T$ for both distributions in the $p=2$ case, with $G_{\max }=10$. It shows that the dispersion of the data increases dramatically as $T$ decreases, especially for
$P_{G}$. Despite the high dispersion, the curve for $P_{G}$ strongly suggests a divergence in the $T \rightarrow 0$ limit, although it seems to be weaker than $\exp (1 / T)$. For $P_{b}$, the presence of frustration causes a much slower increase for both the average of $\xi$ and its dispersion. In fact the curve suggests that it remains finite at $T=0$.

The analysis of the results become more difficult for DHL's with $d_{f}>d_{l c}$, i.e., $p=3$ and 4 . According to renormalization group investigations, a phase transition from the paramagnetic $(P)$ to the SG phase should be observed. ${ }^{14}$ Our results indicate that data scattering and sample average start to increase exponentially at a much higher value of $T$, reaching values as high as $10^{20}$ for $T \simeq 0.25$. The above indicated results for the finite $G_{\max }$ become meaningless.

A simplistic way to address this problem is to increase the value of $G_{\max }$. However, let us note that typical CPU time for performing $\sim 400$ realizations for $\sim 30$ temperature values for $p=3$ and 4 in our present equipment is of the order of 1 day. The magnitude of the data scattering and previous experience in running similar programs for the homogeneous models, indicate that meaningful results should not be expected unless the used value for $G_{\max }$ is significantly increased to $G_{\max }^{\prime}$, so that $G_{\max }^{\prime} / G_{\max } \geqslant 2$. This implies that the CPU time should be increased by a factor $(2 p)^{G_{\max }}$, a limitation that can hardly be met by most of the present day computing systems. To side step this difficulty, we developed an approximate procedure that is able to produce reasonable results, for values of $G>G_{\max }$, with very small increase in CPU time. It amounts to first store $(f, \xi)$ data, for a number $N_{\text {samp }}$ of individually evaluated samples at generation $G_{\max }$, in a data basis $B\left(G_{\max }, N_{\text {samp }}\right)$. Then, use this data and maps (A1)-(A4) to generate another data basis $B\left(G_{\max }\right.$ $\left.+1, N_{\text {samp }}\right)$, with the same number $N_{\text {samp }}$ of samples. Generating new data bases $B\left(G+1, N_{\text {samp }}\right)$ from $B\left(G, N_{\text {samp }}\right)$ can be repeated over and over, for any $G>G_{\max }$, with a very low cost, as the required CPU time increases linearly with $G-G_{\max }>0$. So we return to a iteration scheme that is quite similar to that one used for the homogeneous system. Convergence criteria, similar to the one used in the last case, or a fixed number of iterations can be used to stop the iteration process.

The actual approximation introduced in this scheme refers to the emergence of correlations. Each $G_{\max }$ sample in $B\left(G_{\max }, N_{\text {samp }}\right)$ bares no correlation with any of the other samples, nor within its constituents, as each of its bond was randomly and individually chosen. However, for any $G$ $>G_{\text {max }}$, the same $N_{\text {samp }}$ units will be present in the lattice, in a always larger number, as $G$ increases. Despite the fact that they are randomly distributed, correlation builds up, so that the obtained results constitute approximations to the actual exact scheme, where $G_{\max }$ could be increased without limit.

Results obtained within this approximated scheme for $p$ $=4$ (Gaussian case) are shown in Fig. 6. They were obtained with $N_{\text {samp }}=400$ samples, and convergence criterion is to choose a fixed value $G_{B}=60$. There we draw the quantity $\log _{10}\left(\xi_{L}\right)$, defined as

$$
\log _{10}\left(\xi_{L}\right)=\left\langle\log _{10} \xi\right\rangle
$$




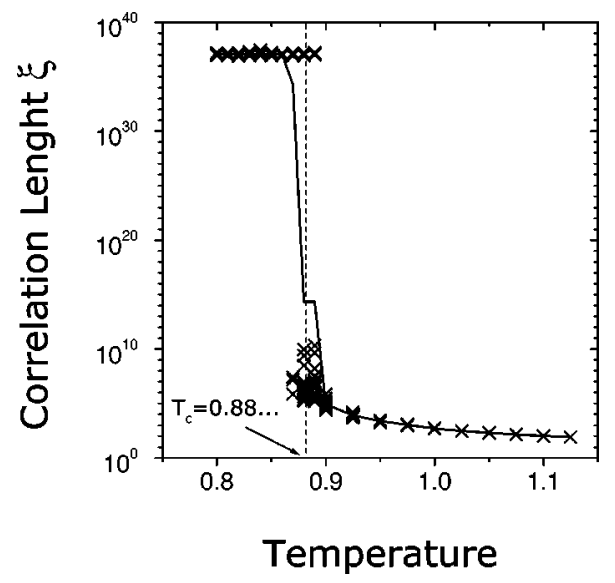

FIG. 6. Log-scale plot of the averaged value (over 400 realizations) of the correlation length $\xi_{L}$ (solid curve) versus temperature for $P_{G}$ for $d_{f}=3$ lattice $(p=4)$. Crosses indicate values for 50 individual realizations for each value of $T$. Large data dispersion is observed only close to the critical temperature, where half of the samples diverge to infinity $\left(\sim 10^{40}\right)$ below $T_{c}$, while others remain finite for $T>T_{c}$.

as function of $T$. We indicate also, for each $T$ value, the individual behavior of the first 50 samples. First, let us discuss the behavior of the individual samples. Much like homogeneous systems, where $\xi$ numerically diverges at the very precise temperature critical value $T_{c}$, we observe that, as $T$ decreases below some value $\left(T_{h i g h}\right)$, some individual samples start to diverge, i.e., $\xi$ jump from values $\leqslant 10^{3}-10^{40}$. The number of samples that so behave increases as $T$ decreases, so that for sufficient low values of $T$ the large majority of samples diverges. This divergent behavior for $\xi$ must be related to the occurrence of a kind of long-range order in the system, hence, of a presumable $P$-SG phase transition. The fact that, for some individual samples, $\xi$ diverges, while for other is it remains finite, is related to the particular set of bonds which was chosen for that sample. It is reasonable to suppose that each particular sample $k$ has its own critical temperature $T_{c, k}$. As the number of divergences increases when $T$ decreases, a value $\bar{T}_{c}$ will be reached when the number of divergences reaches the half of the number of samples. We will consider this value as indicative of the observed phase transition.

Note also that, due to the huge difference in the order of magnitude for $\xi$ when it diverges, a jump in $\langle\xi\rangle$ is indicative of $T_{h i g h}$, but not of $\bar{T}_{c}$. This value can be better evaluated with the help of $\xi_{L}$. If we assume that the numerical value for a divergent $\xi_{D} \sim 10^{R}$, and a typical value for a finite $\xi_{F}$ $\sim 10^{r}$, then $\log _{10}\left(\xi_{L}\right) \sim\left(R N_{D}+r N_{F}\right)$, where $N_{D}$ and $N_{F}$ indicate the number of samples for which $\xi$ diverges and remains finite, respectively. As $N_{D}+N_{F}=N_{\text {samp }}$, it follows that $\log _{10}\left(\xi_{L}\right) \sim\left[N_{D}(R-r)+r N_{\text {samp }}\right]$. Thus, when $N_{D}$ $=N_{\text {samp }} / 2, \log _{10}\left(\xi_{L}\right)$ becomes equidistant to both $\xi_{D}$ and $\log _{10} \xi_{F}$, which is the first criterion for evaluating $\bar{T}_{c}$. The second criterion, which leads to almost the same values, is to look for the inflexion point for the curve $\log _{10}\left(\xi_{L}\right) T$.

Results for $\bar{T}_{c}$ are shown in Table II in comparison to those found in the literature, the latter is obtained by renormalization group schemes.

The presence of correlations induced by the repetition of the same $G_{\max }$ units, for any $G>G_{\max }$, must be the reason for a wide temperature interval above $\bar{T}_{c}$ where some samples have divergent $\xi$. An increase in $G_{\max }$ causes a reduction of the interval $T_{h i g h}-\bar{T}_{c}$. If $G_{\max }$ could be increased without limit, all samples would be free from correlations and the value for $T_{c}$ be well defined.

Estimates for the value of $\nu$ for the four different situations, obtained from the data for $\xi_{L}$, are also indicated in Table II and illustrated in Fig. 7 for $P_{b}$ and $p=4$. Note that our estimates for $\nu$, when $p=3$, are larger than those predicted by RG methods. For $p=4$, however, the obtained value is closer to the one obtained by RG technique, fixing the probability distribution at each renormalization step, ${ }^{14}$ which is a procedure more close to the present approach. On the other hand, the difference is greater when compared with the result obtained by the MKRG scheme where the probability distribution is free to evolve. ${ }^{33}$ The same behavior should occur for the $p=3$ case. It should be noted that in the latter RG analysis, ${ }^{33}$ the value for $\nu$ is not obtained from the direct evaluation of $\xi$, but from a finite-size scaling analysis

TABLE II. Values for the critical temperature $\bar{T}_{c}$ (proportional to the standard deviation of the corresponding distribution) and the correlation length exponent $\nu$ for the Gaussian and $\delta$-bimodal probability distributions,

\begin{tabular}{|c|c|c|c|c|c|c|}
\hline & \multirow[b]{2}{*}{$d_{f}$} & \multirow[b]{2}{*}{$N_{\max }$} & \multicolumn{2}{|c|}{ Critical temperature $T_{C}$} & \multicolumn{2}{|c|}{ Correlation length exponent $\nu$} \\
\hline & & & Gaussian & Bimodal & Gaussian & Bimodal \\
\hline \multirow[t]{4}{*}{ Present work } & $2.585 \ldots$ & 8 & $0.292 \pm 0.001$ & $0.47 \pm 0.007$ & $6.62 \pm 0.15$ & $7.0 \pm 0.2$ \\
\hline & & 9 & $0.297 \pm 0.003$ & $0.49 \pm 0.001$ & $6.57 \pm 0.4$ & $7.0 \pm 0.5$ \\
\hline & 3 & 7 & $0.882 \pm 0.002$ & $1.136 \pm 0.004$ & & $2.4 \pm 0.5$ \\
\hline & & 8 & $0.877 \pm 0.007$ & $1.142 \pm 0.005$ & $2.78 \pm 0.02$ & $2.49 \pm 0.06$ \\
\hline Ref. 14 & 3 & & $0.88 \pm 0.02$ & $1.05 \pm 0.02$ & 2.78 & \\
\hline Ref. 11 & 3 & & $0.88 \pm 0.02$ & $1.05 \pm 0.02$ & 2.809 & \\
\hline Ref. 32 & 3 & & $0.88 \pm 0.05$ & $1.165 \pm 0.01$ & & \\
\hline \multirow[t]{2}{*}{ Ref. 33} & 2.585 & & 0.29 & 0.48 & $2.3 \pm 0.3$ & $2.1 \pm 0.1$ \\
\hline & 3 & & 0.88 & 1.15 & $1.8 \pm 0.2$ & $1.7 \pm 0.1$ \\
\hline Ref. 21 & 3 & & $0.881 \pm 0.001$ & $1.132 \pm 0.001$ & $1.8 \pm 0.1$ & $1.8 \pm 0.1$ \\
\hline
\end{tabular}




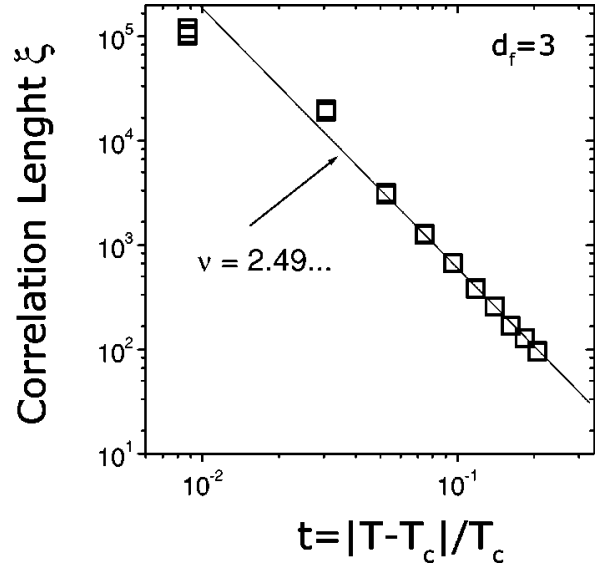

FIG. 7. Evaluation of the critical exponent $\nu$ from the data for $\xi_{L}$, for the $P_{b}$ probability distribution, when $p=4$.

of the behavior of the Edwards-Anderson parameter with respect to the system size, here expressed by the length of the minimal path between the root sites. It should also be noted that the high value of $\nu$, which corresponds to a small value of the thermal exponent, reflects that the system should be close to the marginal behavior ( $d_{f}$ close to $d_{l}$ ).

\section{CONCLUSIONS}

In this work, we proposed an exact scheme for analyzing short-range interaction spin-glasses on hierarchical lattices based on the transfer-matrix formalism, which proved to be quite successful in analyzing both homogeneous and deterministic aperiodic systems. As the scheme explicitly considers the individual contributions of each of the $(2 p)^{G}$ bonds in the generation $G$, the CPU time required for the iteration of the maps which to the thermodynamical properties of the model increases also exponentially with $G$. This makes impossible the iteration of the maps up to the very large values, which have been used in these quoted systems.

Nevertheless, working with the Gaussian and bimodal distributions, we have been able to obtain results for free energy and its derivatives with high degree of accuracy and low dispersion of the data. We have also obtained quantitative agreement for the values of the residual entropies, for the bimodal distribution, with some upper bounds recently obtained by a direct counting of the degeneracy of ground states.

The convergence of $\xi$ presents more difficulties. Already in the uniform systems, its convergence requires larger number of generations. However, this poses a crucial problem for fully disordered systems, as the estimated value of $G$ for the convergence of $\xi$ requires CPU time beyond the capacity of our computing system. We developed an approximate procedure that allows for computing values of $\xi$ for any value of $G$ with low computing cost. However this approximation introduces correlations into the lattice, as the independent $G_{\max }$ units appear several times in the higher generation lattices.

We presented estimations for critical temperature, within the proposed scheme, based on the divergence of the approximate values of $\xi$. We have found that the approximate estimate values for $T_{c}$ are in accordance with those in the literature. We have presented also estimations for critical exponent $\nu$, based on the approximate values for $\xi$.

The nature of the observed transition can be better characterized with a further extension of the proposed scheme. It amounts to evaluating the magnetic properties of the model after the inclusion of a residual external field. This procedure has already been implemented in the case of homogeneous system, and is currently being developed for the disordered case.

\section{ACKNOWLEDGMENTS}

This work was partially supported by the Federal Brazilian granting agencies CNPq, CAPES, and FINEP (under the Grant PRONEX 94.76.0004/97). One of us (S.C.) is also grateful for the financial support received from his local granting agency FACEPE. The authors thank E. Curado and F. D. Nobre for helpful discussions.

\section{APPENDIX A: RECURRENCE MAPS FOR THE FREE ENERGY AND CORRELATION LENGTH EXPONENT FOR THE CASES $p=3$ AND 4}

Let us first introduce a short hand notation with the definition $\overline{z_{G, 2 l}}=z_{G, 2 l} z_{G, 2 l+1}$.

The recurrence equations for $p=3$ are

$$
\begin{aligned}
f_{G+1,0}= & \frac{N_{G}}{N_{G+1}} \sum_{l=0}^{5} f_{G, l}+\frac{T}{N_{G+1}} \\
& \times\left\{\ln [4]-\ln \left[1+\overline{z_{G, 0} z_{G, 2}}+\overline{z_{G, 0} z_{G, 4}}+\overline{z_{G, 2} z_{G, 4}}\right]\right\}
\end{aligned}
$$

and

$$
\begin{aligned}
\frac{1}{\xi_{G+1,0}=} & \frac{M_{G}}{M_{G+1}} \sum_{l=0}^{5} \frac{1}{\xi_{G, l}}+\frac{1}{M_{G+1}} \\
& \times \ln \left[\frac{1+\overline{z_{G, 0} z_{G, 2}}+\overline{z_{G, 0} z_{G, 4}}+\overline{z_{G, 2} z_{G, 4}}}{1+\left(\overline{z_{G, 0} z_{G, 2}}\right)^{-1}+\left(\overline{z_{G, 0} z_{G, 4}}\right)^{1}+\left(\overline{z_{G, 2} z_{G, 4}}\right)^{-1}}\right]
\end{aligned}
$$

For $p=4$, the corresponding equations are

$$
\begin{aligned}
f_{G+1,0}= & \frac{N_{G}}{N_{G+1}} \sum_{l=0}^{7} f_{G, l}+\frac{T}{N_{G+1}} \ln [8]-\frac{T}{N_{G+1}} \ln \left[1+\overline{z_{G, 0} z_{G, 2}}\right. \\
& +\overline{z_{G, 0} z_{G, 4}}+\overline{z_{G, 0} z_{G, 6}}+\overline{z_{G, 2} z_{G, 4}}+\overline{+z_{G, 2} z_{G, 6}}+\overline{z_{G, 4} z_{G, 6}} \\
& \left.+\overline{z_{G, 0} z_{G, 2} z_{G, 4} z_{G, 6}}\right]
\end{aligned}
$$




$$
\begin{aligned}
\frac{1}{\xi_{G+1,0}}= & \frac{M_{G}}{M_{G+1}} \sum_{l=0}^{7} \frac{1}{\xi_{G, l}}+\frac{1}{M_{G+1}} \ln \left[1+\overline{z_{G, 0} z_{G, 2}}+\overline{z_{G, 0} z_{G, 4}}+\overline{z_{G, 0} z_{G, 6}}+\overline{z_{G, 2} z_{G, 4}}+\overline{z_{G, 2} z_{G, 6}}+\overline{z_{G, 4} z_{G, 6}}+\overline{z_{G, 0} z_{G, 2} z_{G, 4} z_{G, 6}}\right] \\
& -\frac{1}{M_{G+1}} \ln \left[1+\left(\overline{z_{G, 0} z_{G, 2}}\right)^{1}+\left(\overline{z_{G, 0} z_{G, 4}}\right)^{-1}+\left(\overline{z_{G, 0} z_{G, 6}}\right)^{-1}+\left(\overline{z_{G, 2} z_{G, 4}}\right)^{1}\right. \\
& \left.+\left(\overline{z_{G, 2} z_{G, 6}}\right)^{-1}+\left(\overline{\left(z_{G, 4} z_{G, 6}\right.}\right)^{-1}+\left(\overline{z_{G, 0} z_{G, 2} z_{G, 4} z_{G, 6}}\right)^{-1}\right] .
\end{aligned}
$$

\section{APPENDIX B: RECURRENCE MAPS FOR THE FIRST DERIVATIVES OF THE FREE ENERGY $f$ AND $z$}

The recurrence maps for the first derivatives of the free energy $f$ and $z$ are

$$
\frac{d f_{G+1,0}}{d T}=\frac{N_{G}}{N_{G+1}} \sum_{l=0}^{3} \frac{d f_{G, l}}{d T}+\frac{1}{N_{G+1}}\left\{\ln 2-\ln \left[1+z_{G, 0} z_{G, 1} z_{G, 2} z_{G, 3}\right]\right\}-\frac{T}{N_{G+1}} \frac{1}{1+z_{G, 0} z_{G, 1} z_{G, 2} z_{G, 3}} \frac{d}{d T}\left\{z_{G, 0} z_{G, 1} z_{G, 2} z_{G, 3}\right\}
$$

and

$$
\frac{d z_{G+1,0}}{d T}=\frac{\left(1-z_{G, 1}^{2} z_{G, 3}^{2}\right) \frac{d}{d T}\left(z_{G, 0} z_{G, 2}\right)+\left(1-z_{G, 0}^{2} z_{G, 2}^{2}\right) \frac{d}{d T}\left(z_{G, 1} z_{G, 3}\right)}{\left(1+z_{G, 0} z_{G, 1} z_{G, 2} z_{G, 3}\right)^{2}} .
$$

For the second derivatives, the corresponding maps are

$$
\begin{aligned}
\frac{d f_{G+1,0}^{2}}{d T^{2}}= & \frac{N_{G}}{N_{G+1}} \sum_{l=0}^{3} \frac{d^{2} f_{G, l}}{d T^{2}}-\frac{2}{N_{G+1}} \frac{1}{1+z_{G, 0} z_{G, 1} z_{G, 2} z_{G, 3}} \frac{d}{d T}\left\{z_{G, 0} z_{G, 1} z_{G, 2} z_{G, 3}\right\} \\
& -\frac{T}{N_{G+1}} \frac{1}{1+z_{G, 0} z_{G, 1} z_{G, 2} z_{G, 3}} \frac{d^{2}}{d T^{2}}\left\{z_{G, 0} z_{G, 1} z_{G, 2} z_{G, 3}\right\}+\frac{1}{N_{G+1}} \frac{1}{\left(1+z_{G, 0} z_{G, 1} z_{G, 2} z_{G, 3}\right)^{2}}\left[\frac{d}{d T}\left\{z_{G, 0} z_{G, 1} z_{G, 2} z_{G, 3}\right\}\right]^{2}
\end{aligned}
$$

and

$$
\begin{aligned}
\frac{d^{2} z_{G+1,0}}{d T^{2}}= & \frac{\left(1-z_{G, 1}^{2} z_{G, 3}^{2}\right) \frac{d^{2}}{d T^{2}}\left(z_{G, 0} z_{G, 2}\right)+\left(1-z_{G, 0}^{2} z_{G, 2}^{2}\right) \frac{d^{2}}{d T^{2}}\left(z_{G, 1} z_{G, 3}\right)}{\left(1+z_{G, 0} z_{G, 1} z_{G, 2} z_{G, 3}\right)^{2}} \\
& -2 \frac{z_{G, 1} z_{G, 3}\left(1-z_{G, 1}^{2} z_{G, 3}^{2}\right)\left[\frac{d}{d T}\left(z_{G, 0} z_{G, 2}\right)\right]^{2}+z_{G, 0} z_{G, 2}\left(1-z_{G, 0}^{2} z_{G, 2}^{2}\right)\left[\frac{d}{d T}\left(z_{G, 1} z_{G, 3}\right)\right]^{2}}{\left(1+z_{G, 0} z_{G, 1} z_{G, 2} z_{G, 3}\right)^{3}} \\
& -4 \frac{\left(z_{G, 0} z_{G, 2}+z_{G, 1} z_{G, 3}\right)\left[\frac{d}{d T}\left(z_{G, 0} z_{G, 2}\right)\right]\left[\frac{d}{d T}\left(z_{G, 1} z_{G, 3}\right)\right]}{\left(1+z_{G, 0} z_{G, 1} z_{G, 2} z_{G, 3}\right)^{3}} .
\end{aligned}
$$

*Electronic address: randrade@ufba.br

†Electronic address: enogue@ufba.br

Electronic address: sergio@1ftc.ufpe.br

${ }^{1}$ K. Binder and A.P. Young, Rev. Mod. Phys. 58, 801 (1986).

${ }^{2}$ K.H. Fischer and J.A. Hertz, Spin Glasses (Cambridge University Press, Cambridge, 1991).

${ }^{3}$ M. Mézard, G. Parisi, and M.A. Virasoro, Spin Glass Theory and Beyond (World Scientific, Singapore, 1987).

${ }^{4}$ Spin Glasses and Random Fields, edited by A.P. Young (World Scientific, Singapore, 1998).
${ }^{5}$ E. Marinari, G. Parisi, F. Ricci-Tersenghi, J.J. Ruiz-Lorenzo, and F. Zuliani, J. Stat. Phys. 98, 973 (2000)

${ }^{6}$ W.L. McMillan, J. Phys. C 17, 3179 (1984); A.J. Bray and M.A. Moore, Phys. Rev. B 31, 631 (1985); Phys. Rev. Lett. 58, 57 (1987); D.S. Fisher and D.A. Huse, ibid. 56, 1601 (1986); Phys. Rev. B 38, 386 (1988).

${ }^{7}$ For a recent topical review on this issue see C.M. Newman and D.L. Stein, cond-mat/0301403 (unpublished).

${ }^{8}$ For a review, see H. Rieger, Frustrated Systems: Ground State Properties via Combinatorial Optimization, Lecture Notes in 
Physics Vol. 501 (Springer-Verlag, Heidelberg, 1998); See also N. Kawashima and H. Rieger, Europhys. Lett. 39, 85 (1997).

${ }^{9}$ A.K. Hartmann, Europhys. Lett. 40, 429 (1997); 44, 249 (1998); A.K. Hartmann, Phys. Rev. B 59, 3617 (1999); Phys. Rev. E 59, 84 (1999).

${ }^{10}$ B.A. Berg, U.E. Hansmann, and T. Celik, Phys. Rev. B 50, 16444 (1994).

${ }^{11}$ M. Mézard and G. Parisi, Eur. Phys. J. B 20, 217 (2001).

${ }^{12}$ R.R.P. Singh and S. Chakravarty, Phys. Rev. Lett. 57, 245 (1986); L. Klein, J. Adler, A. Aharony, A.B. Harris, and Y. Meir, Phys. Rev. B 43, 11249 (1991).

${ }^{13}$ R.N. Bhatt and A.P. Young, Phys. Rev. Lett. 54, 924 (1985); A.T. Ogielski and I. Morgenstern, ibid. 54, 928 (1985); R.N. Bhatt and A.P. Young, Phys. Rev. B 37, 5606 (1988).

${ }^{14}$ B.W. Southern and A.P. Young, J. Phys. C 10, 2179 (1977); N. Kawashima and A.P. Young, Phys. Rev. B 53, R484 (1996).

${ }^{15}$ A.A. Migdal, Zh. Eksp. Teor. Fiz. 69, 810 (1975) [Sov. Phys. JETP 42, 413 (1976)]; 69, 1457 (1975) [42, 743 (1976)].

${ }^{16}$ L.P. Kadanoff, Ann. Phys. (N.Y.) 100, 359 (1976).

${ }^{17}$ G. Forgácz and A. Zawadowski, Acta Phys. Acad. Sci. Hung. 42, 353 (1977).

${ }^{18}$ A.N. Berker and S. Ostlund, J. Phys. C 12, 4961 (1979).

${ }^{19}$ P.M. Bleher and E. Zälys, Commun. Math. Phys. 67, 17 (1979).

${ }^{20}$ S. Prakash and I.A. Campbell, Physica A 235, 507 (1997).

${ }^{21}$ E. Nogueira, Jr., S. Coutinho, F.D. Nobre, and E.M.F. Curado, Physica A 271, 125 (1999).

${ }^{22}$ E.M.F. Curado, F.D. Nobre, and S. Coutinho, Phys. Rev. E 60, 3761 (1999).
${ }^{23}$ Fernando D. Nobre, Physica A 280, 456 (2000).

${ }^{24}$ M.A. Moore, Herman Bokil, and Barbara Drossel, Phys. Rev. Lett. 81, 4252 (1998); Barbara Drossel, Herman Bokil, M.A. Moore, and A.J. Bray, Eur. Phys. J. B 13, 369 (2000); Herman Bokil, Barbara Drossel, and M.A. Moore, Phys. Rev. B 62, 946 (2000); Barbara Drossel and M.A. Moore, Eur. Phys. J. B 21, 589 (2001); Barbara Drossel, Herman Bokil, and M.A. Moore, Phys. Rev. E 62, 7690 (2000).

${ }^{25}$ S.T.R. Pinho, T.A.S. Haddad, and S.R. Salinas, Physica A 257, 515 (1998); A.C.N. Magalhães, S.R. Salinas, and C. Tsallis, J. Phys. A 31, L567 (1998); T.A.S. Haddad, S.T.R. Pinho, and S.R. Salinas, Phys. Rev. E 61, 3330 (2000); E. Nogueira, Jr., R.F.S. Andrade, and S. Coutinho, Eur. Phys. J. B 23, 373 (2001).

${ }^{26}$ S. Franz, G. Parisi, and M.A. Virasoro, J. Phys. (France) 4, 1657 (1994).

${ }^{27}$ R. Rajiv, P. Sing, and M.E. Fischer, J. Appl. Phys. 63, 3994 (1988).

${ }^{28}$ O.D. da Silva-Neto, DSc. thesis, Universidade Federal de Pernambuco (1999).

${ }^{29}$ R.F.S. Andrade, Phys. Rev. E 59, 150 (1999).

${ }^{30}$ A. Crisanti, G. Paladin, and A. Vulpiani, Products of Random Matrices in Statistical Physics, Spinger Series in Solid State Sciences Vol. 104 (Springer-Verlag, Berlin, 1993).

${ }^{31}$ I. Morgenstern and K. Binder, Phys. Rev. B 22, 288 (1980).

${ }^{32}$ L.W. Bernardi, S. Prakash, and I.A. Campbell, Phys. Rev. Lett. 77, 2798 (1996).

${ }^{33}$ E. Nogueira, Jr., S. Coutinho, F.D. Nobre, and E.M.F. Curado, Physica A 257, 365 (1998). 